\title{
XIV. An account of experiments for determining the length of the pendulum vibrating seconds in the latitude of London
}

\section{Capt. Henry Kater F.R.S.}

To cite this article: Capt. Henry Kater F.R.S. (1818) XIV. An account of experiments for determining the length of the pendulum vibrating seconds in the latitude of London, Philosophical Magazine Series 1, 52:244, 90-100, DOI: 10.1080/14786441808652012

To link to this article: http://dx.doi.org/10.1080/14786441808652012

曲 Published online: 23 Jul 2009.

Submit your article to this journal $\pi$

山 Article views: 2

View related articles $\sqsubset$ 
90 An Account of Experiments for delermining the Length of

think proper to dispute the accuracy of my foregoing computation, or the justice of my disapproval, (and that you will be pleased to afford me your pages as the medium,) I shall enter without hesitation into all the minutiæ of the question.

\section{I am, sir,}

Your most oluedient servant,
St. Finbars, Cork, July 15, 1818.
William Gutteridge.

XIV. An Account of Experiments for determining the Length of the Pendulum vibrating Seconds in the Latitude of London. By Capt. Henry Kater, F.R.S.*

\section{$\mathbf{T}$}

Tetermine the distance between the point of suspension and centre of oscillation of a pendulun vibrating seconds in a given latitude, has long been a desideratum in science. Many experiments have been made for this purpose ; but the attention of all who have hitherto engaged in the inquiry (excepting Whitehurst) appears to have been directed to the discovery of the centre of oscillation. The solution of this problem depending, however, on the uniform density and known figure of the body employed, (requisites difficult if not impossible to be ensured in practice,) it is not surprising that the experiments made by different persons should have been productive of various results.

When I had the honour of being appointed one of the committee of the Royal Society for the investigation of this interesting subject, I imagined that the least objectionable mode of proceeding would be to employ a rod drawn as a wire, in which, supposing it to be of equal density and diameter throughout, the centre of oscillation, as it is well known, would be very nearly at the distance of two-thirds of the length of the rod from the point of suspension; and I purposed by inverting the rod, and taking a mean of the results in each position, to obviate any error which might arise from a want of uniformity in density or figure. After numerons trials however, and as frequent disappointments, I was at length convineed of the impracticability of obtaining a rod sufficiently uniform; and I was besides aware, that under certain circumstances errors might arise from this cause which it would be impossible by any method to detect.

Not feeling at all satisfied with the prospect which the use of a rod presented, I endeavoured to discover some property of the pendulum of which I might avail myself with greater probability of success; and I was so fortunate as to perceive one, which

* From the Transactions of the Royal Society for 1818, part $i$. 


\section{the Penduhum vilrating Seconds in the Latitude of London. 91}

promised an unexceptionable result. It is known that the centres of suspension and oscillation are reciprocal; or, in other words, that if a body he suspended by its centre of oscillation, its former point of suspension becomes the centre of oscillation, and the vibrations in both positions will be performed in equal times. Now the distance of the centre of oscillation from the point of suspension, depending on the figure of the body employed, if the arrangement of its particles be changed, the place of the centre of oscillation will alsu suffer a change. Suppose then a body to be furnished with a point of suspension, and another point on which it may vibrate, to be fixed as nearly as can be estimated in the centre of oscillation, and in a line with the point of suspension and centre of gravity. If the vibrations in each position should not be equal in equal times, they may readily be made so, by shifting a moveable weight, with which the body is to be furnished, in a line between the centres of suspension and oscillation; when the distance between the two points about which the vibrations were performed being measured, the length of a simple pendulum and the time of its vibration will at once be known, uninfluenced by any irregularity of density or figure*.

An unexceptionable principle being thus adopted for the con-

* In the Conmoissance des Temps for 1820, is an article by M. de Prony on a new method of regulating clocks. At the conclusion of this article is a short note, in which the author adds, "J'ai propose en 1790 à l'Academie des Sciences un moyen de déterminer la longueur du pendule en faisant osciller un pendule composé sur deux ou trois axes attachés à ce corps. (yoyez mes Leçons de Mécanique, art. 1107 et suivans.) Il paroit qu'on a fait ou qu'on va faire usage de ce moyen en Angleterre." On referring to the Lecons de Mécanique, as directed, I can perceive no hint whatever of the possibility of determining the length of the seconds pendulum by means of a compound pendulum vibrating on two axes; but it appears that the method of M. de Prony consists in employing a compound pendulum having three fixed axes of suspension, the distances between which, and the time of vibration upon each, being known, the length of three simple equivalent pendulums may thence be calculated by means of formulæ given for that purpose. M. de Prony indeed proposes employing the theorem of Huygens, of which $I$ have availed myself, of the reciprocity of the axis of suspensien and that of oscillation, as one amongst other means of simplifying his formulæ, and says, " J'ai indiqué les moyens de concilier avec la condition à laquelle se rapportent ces formules, celle de rendre l'axe moyen le recipreque de l'un des axes extrêmes; J'emploie pour les ajustemens qu'exigent ces diverses conditions un poids curseur dont j’ai exposé les propriétés dans un mémoire publié avec la Connoissance des Temps de 1817." Now it appears evident from this passage, that $M$. de Prony viewed the theorem of Huygens solely with reference to the simplification of his formula; for, had he perceived that he might thence have obtained at once the length of the pendulum without further calculation, the inevitable conclusion must instantly have followed, that his third axis and his formulæe were wholly unneecssary. 
struction of the pendulum, it became of considerable importance to select a mode of suspension equally frce fron objection. Diamond points, spheres, and the knife edge, were each considered; but as it was found difficult to procure diamond points sufficiently well executed, the knife edge was preferred, after many experiments had been made with spheres, the result of which it may not be useless for a moment to dwell upon.

It is known, that if two curved surfaces be ground together in every possible direction, they will become portions of spheres; and thus a perfect sphere may be formed by grinding a ball in a hemispherical cup. If a peudulum vibrate on surh a sphere, working in a conical aperture, it is evident that the centre of the sphere will be accurately in the axis of vibration. In aring this method, however, it was found that the friction was so considerable, as to bring the pendulum to a state of restafice a fex: vibrations ; and when the friction was suficientiy diminished, by a contrivance which it is unnecessary to describe, tise lateral force of the pendulum in an arc of two degrees and a half, was stfficiently powerful to carry the ball entirely out of the socket; and it was consequently evident, that though the arc of vibratiou might not be large enough to effect this, it must necessarily cause the ball in some degree to ascend the inclined plane of the aperture; and this consideration induced me to abandon at once a mode of suspension which I should otherwise have esteemed the best that coukl have been employed.

The principal objections to the use of a knife edge, appeared to be, the rifficulty of forming it perfectly straight, and the possibility that it might suffer a change of figure from wear, during the experiments, which might introduce an error not to be detected. The first of these objections I found to be perfectly groundless, as a knife edge can be made so as not to deviate sensibly from a right line. The second objection would indeed be of weight, were the usual method of determining the time of vibration resorted to, by comparing the pendulum with a clock, at the distant intervals of 24 hours ; but it will hereafter appear, that should any alteration in the form of the knife edge take place, it must become perceptible every ninth minute ; in addition to which, I proposed to measure the distance of the knife edges both before and after the experiments, when any change would of course be immediately detected.

\section{Description of the Pendulum employed.}

The pendulum constructed upon these principles is formed of a bar of plate brass, one inch and a half wide, and one eighth of an inch thick. Through this bar, two triangular holes are made, at the distance of $39 \cdot 4$ inches from eacli other, to admit 
the knife edges. Four strong knees of hammered brass of the same width as the bar, six inches loug and three quarsers of an inch thick, are firmly screwed by pairs to each end of the bar, in such a manner, that when the knife edges are passed through the triangular apertures, their backs mav bear steadily against the perfectly plane surfaces of the brass knees, which are formed as nearly as possible at right angles to the bar. The bar is cut. of such a length, that its ends may be short of the extremicies of the knee pieces about two inches.

Two slips of deal 17 inches long, and of the same thickness as the bar, are inserted in the spaces thus left between the knee pieces, and are firnly secured there by pins and screws. These slips of deal are oniy half the width of the bar; they are stained black, and in the extremity of each a small whalebone point is inserted, for the purpose of indicating the extent of the arc of vibration.

A cylindrical weight of brass, three inches and a half diameter, one inch and a quarter thick, and weighing about two pounds seven ounces, has a rcctangular opening in the direction of its diameter, to adrit the knee pieces of one end of the pendulum. This weight being passed on the pendulum, is so thoroughly secured there by means of a conical pin fitting an opening made through the weight and knee pieces, as to render any change of position impossible. A second weight, of about seven ounces and a half, is made to slide on the bar near the knife edge at the opposite end; and this weight may be fixed at any distance on the bar by two screws with which it is furnished.

$A$ third weight, or rather slider, of only four ounces, is moveable along the bar, and is capable of nice adjustment by means of a screw fixed to a clamp, which clamp is included in the weight. This slider is intended to move near the centre of the bar. It has an opening, through which may be seen divisions, each equal to one-twentieth of an inch, engraved on the bar; and a line is drawn on the edge of the opening to serve as an index to determine the distance of the slider from the middle of the bar.

We now come to the most important part, the knife edges. These are made of that kind of steel which is prepared in India, and known by the name of wootz. Their form is triangular, and their length one inch and three quarters. Mr. Stodard was so obliging as to forge them for me: they were made as hard as possible, and tempered by immersing them merely in boiling water.

The knife edges were ground on a plane tool, which necessarily ensured a perfectly straight edge. This was ascertained by bringing the edge of the che in contaet with the plane of the other, 


\section{An Account of Experiments for determining the Length of}

other, when, if no light was perceptible between them in any position, it was inferred that the edge was a right line. They were then earefully finished on a plane green hone, giving them such an inclination as to make the angle on which the vibrations are petformed about 120 degrees.

Previously to the knife edges being hardened, each was tapped half way through, near the extremities, to receive two screws, which being passed through the knee pieces, drew the knife edges into close contact with them, the surfaces of both having been previously ground together to guard against any strain which might injure their figure.

\section{The Support, and other Apparatus.}

The support of the pendulum consists of a piece of bell metal six inches long, three inches wide, and three-eighths of an inch thick. An opening is made longitudinally through half the length of the piece, to admit the peudulum, and the bell metal is cast with a rectangular elevation on each side of the opening extending the whole length of the piece. Two plates of agate* were cemented to this elevated part, beds having been made to receive them, in order that their surfaces might be in the same plane with that of the bell metal. The whole was then ground perfectly flat. A frame of brass is attached by two opposite screws, which serve as centres, to the sides of the elevated part of the support; and one end of this frame being raised or depressed by means of a screw, the pendulum when placed with its knife edge resting in $\mathrm{Ys}$, at the other end of the frame, could be elevated entirely above the surface of the agate, or be gently lowered until the knife edge rested wholly upon it; and thus the knife edge was sure to bear always precisely on the same part of the agate plane, by elevating the Ys above its surface, placing the knife edge in them, and then letting down the whole gently by means of the serew, till the Ys were completely clear of the knife edge. The support was firmly screwed to a plank which will hereafter be described.

To the kindness of Henry Browne, Esq. F.R.S., I am essentially indebted for the success of the experiments which form the subject of this paper. He most obligingly allowed me the use of his house, his excellent time-pieces, and transit instrument, assisting me with indefatigable zeal by his very accurate daily observations, and intermediate comparisons for determining the rate of the clock. The house is substantially built, and is situ-

* Plates of hard steel were first tricd, but werc found to have suffered penetration by the knife edge. 
ated in a part of Portland Place not liable to much disturbance from the passing of carriages. The room in which the experiments were made is the last of two on the ground floor, communicating with each other and facing the north. The temperature consequently is very steady, and, if necessary, may be raised to any given degree by a fire in the first room. The clock with which the pendulum was compared was made by Arnold; and in addition to the gridiron compensation for temperature, its pendulum is suspended by a spring, the strength of which is so adjusted, that the vibrations in different arcs are performed in equal times. This clock is firmly screwed to the wall, in a recess opposite to the window. Near to this, on the wall which is at right angles to the recess, is fixed another time-piece by Cumming, which was the property of the late General Roy, and is considered by $\mathrm{Mr}$. Browne to be the best in his possession. Respecting this clock, it will be sufficient to remark, that threetenths of a second was the greatest variation in its daily rate from the 22d February, when the observations commenced, to the 31 st July; and consequently the deviation from its mean rate during that period, did not exceed $0 \cdot 15$ of a second per day. This clock has been used as the standard of comparison, the time having been taken from the transit instrument by a chronometer of Arnold's. With such advantages it will be confessed that there can be little chance of error arising from the rate of the clock.

A plank of well seasoned mahogany, two feet wide, and three inches thick, was forcibly driven between the walls forming the sides of the recess, until it was near the top of the clock case. To this the support of the pendulum before described was firmly screwed, and carefully levelled, in such a position as to allow the pendulum to vibrate as near as possible to the clock-case without touching it; and that, when at rest, it might appear to an observer in front of the clock, to pass over the centre of the dialplate, its extremity reaching a little below the centre of the ball of the pendulum. Beneath, fixed to the clock-case, was an are divided into degrees and tenths, to determine the extent of the vibrations. Such a portion of the plank was cut away as was necessary to admit of the pendulum being placed on its support. A circular white disk was pasted on a piece of black paper, which was attached to the ball of the pendulum of the clock; and this disk was of such a diameter, as, when both pendulums were at rest, to be just hid from an observer at the ppposite side of the room, by one of the slips of deal which form the extremities of the brass pendulum.

Though there was little reason to imagine that the vibrations of the pendulum could communicate any motion to a support so 


\section{An Account of Experiments for determining the Length of}

firm as that which has been described, it became a point of considerable importance to verify this by actual experiment. For this purpose 1 had recourse to a delicate and simple instrument invented by Mr. Hardv, elock-maker, the sensibility of which is such, that har the slightest motion taken place in the support, it must have been instantly deteeted. This little instrument consists of a steel wire, the lower part of which inserted in the piece of brass which serves as its support, is flattened so as to form a delicate spring. On the wire, a small weight slides, by means of which it may be made to vibrate in the same time as the pendulum to which it is to be applied as a test. When thus adjusted, it is placed on the material to which the pendulum is attached; and should this not be perfectly firm, it's motion will be commmicated to the wire, which in a little time will accompany the pendulum in its vibrations. This ingenious contrivance zappeared fully adequate to the purpose for which it was employed, and afforded a satisfactory proof of the stability of the point of suspension.

$\Lambda$ firm triangular wooden stand, as high as the ball of the pandhum, was screwed to the floor at the distance of nine feet in front of the clock. This served as a support, to which was attached a small telescope, magnifving about four times, which was capable of a horizontal motion on its axis, a vertical motion, and a motion at right angles to the line of sight. In the focus of the eye-glass was a diaphragin forming a perpendicular opetning, the sides of which were parallel, and capable of being piaced nearer, or further asunder. The edges of this diaphragm were adjusted so as to form tangents to the horizontal diameter of the white disk, and consequently to coincide with the edges of the slip of deal. When, therefore, both pendulums were at rest, nothing was visible through the telescope, excepting the divided are for ascertaining the extent of the vibrations, and which was seen through a horizontal opening made for that purpose in the top of the diaphragm.

\section{Methad of determining the Number of Viltrations made by the Pendulum in twenty-four IJours.}

If both pendulums be now set in motion, the brass pendulum a little preceding that of the clock, the following appearances nay be remarked. The slip of deal will first pass through the field of view of the telescope at each vibration, and will be followed by the white disk. But the distance between the centres of suspension and oscillation in the brass pendulum being rather the longer, the pendulum of the clock will gain upon it, the white hisk will gradually approach the slip of deal, and at leugth, at a certain vibration, will be wholly concealed by it. The minute 
and second at which this total disappearance is observed, must he noted. The pendulums will now be seen to separate, and after a time will again approach each other, when the same phænomenon will take place. The interval between the two coincidences in seconds, will give the number of vibrations made by the pendulum of the elock; and the number of oscillations of the brass pendulum, in the same interval, may be known by considering that it must have made two oscillations less than the pendulum of the clock. Hence by simple proportion, as the vibrations made by the pendulum of the clock are to the number of vibrations made by the brass pendulum, so are the vibrations made by the pendulum of the clock in 24 hours, to those of the brass pendulum in the same period*.

Many experiments were made in order to select such a distance of the knife edges as might give an interval which would allow of the determination of the time of coincidence without an error of a single second $t$, and yet afford a convenient number of intervals before it should become necessary to renew the motion of the pendulum. At the first coincidence, the velocity of the brass pendulum, at the lowest part of the arc, must not exceed that of the pendulum of the clock, otherwise the disk would disappear for an imperceptible time, and then re-appeạr; and this limits the extent of the arc of vibration.

Again; the observations must not be continued beyond a certain diminution of the arc of vibration; otherwise the space, which the pendulum of the clock has to gain on the brass pendulum in one vibration, becomes so small as to render the observation of the time of coincidence in some degree uncertain; and, should the space be so far diminished as to be less than the error or deviation from a right line, which would probably take place in the atjustment of the sides of the diaphragm, the end of the pendulum, and the disk, the results would be erroneous, as the interval would go on increasing till the pendulum came to a state of rest.

The interval which best fulfilled these conclitions was found to be about 530 seconds. This admitted five coincidences (affording four intervals) to be taken before the are became too small for the observations to be continued with safety. With this interval an error of one second in the time of coincidence

\footnotetext{
* In order to render the calculation more easy, the clock has always been supposed to keep mean time, or to make 86,400 vibrations in 24 hours, and the variation from this number, or the rate of the clock (being a very small quantity) has been afterwards applied as a correction.

+ The principle on which this method of coincidences is founded, was employed by Dr. Wollaston, in May 1808, in some experiments in which he was then engaged, the moment of coincidence being determined however by sound instead of sight.

Vol. 52. No. 244. Aug. 1818. 


\section{An Account of Experiments for determining the Length of}

would occasion an error of only 0.63 in the number of vibrations in 24 hours.

Here it must be evident that no sensible alteration could take place in the knife edge during the experiments withoet its becoming perceptible at every eoincidence, since the number of vibrations in 24 hours deduced from each interval, must vary with any change in the form of the knife edge.

The following was the method pursued in making the observations. The small weight or slider being placed with its index at a certain distance (say one inch and a half) from the middle of the pendulum towards the great weight, and the second weight about five inches from the knife edge, the Ys of the support were elevated, the knife edge of the pendulum was placed in them, with the great weight above, and the frame gently lowered till the knife edge was left on the surface of the agate. The requisite adjustments of the telescope having been made, the pendulum was sct in motion in an are not exceeding one degree and four-tenths, in order that its velocity might not be greater than that of the pendulum of the clock.

The minute and second, at which the disk ceased to be visible, was then carefully noted; and the arc of vibration seen through the telescope, the height of the barometer, and the temperature indicated by a thermometer suspended on the clock-case near the middle of the brass pendulum, were also observed and registered. Five suecessive coincidences were thus taken, and the number of vibrations in 24 hours was deduced from them in the manner before described; but the vibrations thus obtained being made in different ares, it became necessary to apply a correction to determine what they would have been in an arc infinitely small. For this correction I might have used a formula depending on the decrease of the arcs in geometrical progression, whilst the times decrease in arithmetical; but as there is an uncertainty in observing the arc of vibration amounting to one or two hundredths of a degree, this method, though more perfect in theory, would have been an unnecessary refinement in practice.

The error arising from the greater length of the vibration in a circular arc, being nearly as the square of the arc, if the mean of the observed arcs at the commencement and end of each interval be taken, and its square multiplied by $1 \cdot 635$, (the difference between the number of vibrations made by the pendulum in 24 hours, in a cycloid and in an arc of one degree,) the required correction will be obtained, to be added to the number of vibrations before computed.

The mean of these last results being taken, and also the mean of the observed temperatures at the first and last coincidences, the number of vibrations in 24 hours was obtained at a certain temperature, 
temperature, and altitude of the barometer, in an infinitely small arc, the great weight being alove.

The frame of the support was now elevated, the pendulum was inverted, placed in the $Y \mathrm{~s}$, with the great weight below; and the knife edges being gently let down as before on the agate plane, the same process with respect to the observations was followed, which has just been described. And if the mean temperature differed from that in the former position of the pendulum, the mean number of vibrations was corrected for such difference of temperature, the expansion of the pendulum being linown by experiments hereafter to be detailed, and consequently the gain or loss in 24 hours by a given change of temperature.

The mean number of vibrations thus found, differing from that given in the former position of the pendulum, the second weight was moved, the number of vibrations again determined; and the pendulum being iuverted, the process was repeated until the vibrations in 24 hours, in either position of the pendulum, were brought as near to an equality as could readily be effected by means of this weight: it was then firmly secured in its place.

Whatever alteration may be made in the arrangement of the weights, the effect cn the vibrations (except in one particular instance) will be the same in both positions of the pendulum, always increasing or diminishing their number in both cases, though in different degrees; and the vilrations will be least affected ly such change when the great weight is below, and will consequently be nearest to the truth in this position. No donbt, therefore, can arise, as to the kind of correction required. The number of vibrations after the adjustment by the second weight has been completed, must be left in defect, for a reason which will be immediately apparent.

There is a point in the pendulum where the effect of the slider in increasing the number of vibrations is a maximum; and it appears from Dr. Young's investigations, that this point in one position of the pendulum is different from that in the other. Very near either of these points, the pendulum being in its corresponding position, the motion of the slider produces scarcely any change in the number of viorations; but the slider being then more distant from the point of maximum belonging to the other position of the pendulum, the corresponding increase of the number of vibrations arising from such motion of the slider, will in that position be very perceptible.

In the present instance, the point of maximum, in either position of the pendulum, is abont four-tenths of an inch below the middle, and consequently the distance of the two points from each other is ahout eight-tenths of an inch. 'The slider, which had. remained stationary during the adjustment of the sccond G 2 weight 
weight at about one inch and a half from the millile of the pendulum towards the great weight, must now be shifted (say one inch) towards the middle of the pendulum, in order to increase the number of vibrations which it may be recollerted were left in defect, so that they may be in excess. It is evident that the true number of vibrations will be found, when the slider is somewhere between its first and second position. Let the slider be now placed half-way between these two points. If the number of vibrations in this third position be still in excess, the truth will lie between the first and third positions of the slider. And thus, by continually bisecting with the slider, the distance of the two last found points, the number of vibrations when the great weight is lelow', will rapidly approach the truth, being alternately in defect and in excess; and when the approximation is such as that the difference in either position of the pendulum becomes inconsiderable, the vibrations, when the great weight is below, may be taken for the truth; and thus the number of vibrations in 24 hours, of a pendulum equal in length to the distance between the knife edges, will be known at a certain temperature, and at an observed height of the barometer.

[To be continued.]

XV. Account of Experiments made by the Assay Master of the King of the Netherlands, at the Mint of Utrecht, on the Native Copper existing in Blocks on the South Side of Lake Superior, communicated by a Letter from Mr. Eustis, Minister Plenipotentiary and Envoy Extraordinary from the United States, Esc. to Dr. Sa muel L. Mrtchrlu, dated Hague, Oct. 12, 1817.

Dear Sir, - Perceiving by the public newspapers, that my friend Dr. Le Barron had presented you a piece of copper, I inclose the analysis of a piece which he gave me at the mint of Utrecht, a portion of which, in its crude state, I presented to the minister of foreign affairs, to be deposited in the university of Iseyden. My object in procuring an assay in a foreign country, was first to add to the diffusion of information respecting our country; and secondly, that it might be compared with experiments made in the United States. I had hoped to return this autumn, and to have taken it with me; but the state of our commercial relations with this country has necessarily deferred that hope until the spring. I $\mathrm{nm}, \& \mathrm{c}$.

The Hon. Samuel L. Mitchill.

W, Eustis. 